\title{
Analysis of Noise-Injection Networks for Interferometric-Radiometer Calibration
}

\author{
Ignasi Corbella, Member, IEEE, Adriano Camps, Associate Member, IEEE, Francesc Torres, and Javier Bará
}

\begin{abstract}
The spatial resolution of current space-borne Earth observation radiometers is limited by the physical antenna aperture. This is especially critical at $L$-band, which exhibits high sensitivity to soil moisture and sea surface salinity. Interferometric radiometers (InR's) are currently being studied by several space agencies as a feasible alternative to overcome this problem. However, their calibration is a crucial issue since most techniques inherited from radio astronomy cannot be directly applied. Due to the large number of receiving channels, calibration techniques based on centralized noise injection from a single noise source will require a large and stable distribution network, which is technically very complex and unacceptable from the point-of-view of mass and volume. Procedures based on distributed noise injection from a set of noise sources through smaller distribution networks have been recently proposed by the authors as an alternative to alleviate these technological problems. In this paper, the analysis of these networks, the impact of the noise generated by the network losses on the calibration, and the impact of front-end reradiated noise are analyzed. Finally, the optimum circuit topologies and tolerances to which these networks have to be characterized in order to achieve the required calibration are derived. These configurations are formed by cascading basic $1: 2$ and $1: 3$ isolated power splitters. Isolators at receivers' inputs have to be included in order to minimize offsets originated from the correlation of reradiation of receiver noise. It has been found that, in order to satisfy the calibration requirements of InR's, the $S$-parameters of the ensemble noise-injection network plus isolators have to be known to within $0.025-0.050 \mathrm{~dB}$ in amplitude and $0.5^{\circ}$ in phase, and their physical temperature known to within $0.5^{\circ} \mathrm{C}$.
\end{abstract}

Index Terms-Calibration, interferometry, noisy networks, radiometry.

\section{INTRODUCTION}

D ERIVED measurements of soil moisture and sea surface salinity can be performed by means of passive observations at $L$-band. At present, the global coverage requirements, the low revisit time (1-3 days), the radiometric resolution $(1 \mathrm{~K})$ and the spatial resolution $(\sim 30 \mathrm{~K} \cdot \mathrm{m})$ can be achieved by means of low Earth orbit interferometric radiometer (InR). The basic measurement of an InR is the so-called visibility function $V_{k j}$ (units of kelvin) obtained from the complex cross correlation of the signals $b_{k 2}(t)$ and $b_{j 2}(t)$ collected by each pair of antennas

Manuscript received December 2, 1998. This work was supported by the Spanish Comisión Interministerial de Ciencia y Tecnología under Grant CICYT TIC 96/0879, and by the European Space Agency MIRAS Calibration System Definition CAS-D Project under ESTEC Contract 12513/97/NL/MV.

The authors are with the Universitate Politècnecia de Catalunya, 08034

Barcelona, Spain (e-mail: corbella@tsc.upc.es).

Publisher Item Identifier S 0018-9480(00)02782-4.

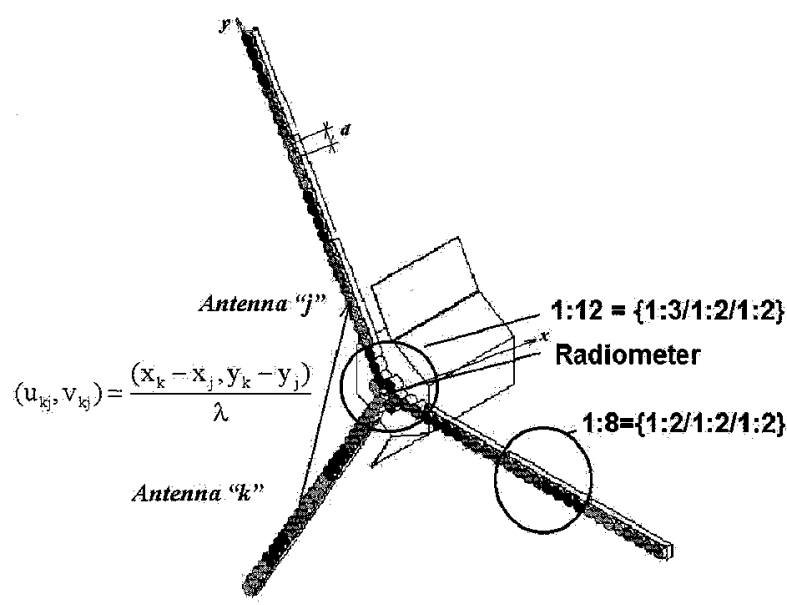

Fig. 1. Diagram of a $Y$-shaped InR similar to MIRAS, but with 27 antennas per arm. Groups of four antennas have been shaded to differentiate them from the rest. The three extra antennas in the center are calibrated by noise injection, and are used for calibration by means of phase/amplitude closures. Each antenna pair leads to a visibility sample $V_{k, j}\left(u_{k, j}, v_{k, j}\right)=1 / 2\left\langle b_{k: 2}(t) b_{j 2}^{*}(t)\right\rangle$. The antenna in the center is connected to a radiometer that measures the zero baseline $V(0,0)=T_{A}$.

$k$ and $j$ (Fig. 1). The antennas are located in the $X Y$-plane and spaced a normalized distance $\left(u_{k j}, v_{k j}\right)=\left(x_{j}-x_{k}, y_{j}-\right.$ $\left.y_{k}\right) / \lambda_{0}$, which is called the baseline [1], [2] or the spatial frequency being sampled as follows:

$$
\begin{aligned}
V_{k j}= & V\left(u_{k j}, v_{k j}\right) \\
\triangleq & \frac{1}{k_{B} \sqrt{B_{k} B_{j}} \sqrt{G_{k} G_{j}}} \cdot \frac{1}{2}\left\langle b_{k 2}(t) b_{j 2}^{*}(t)\right\rangle \\
\triangleq & \frac{1}{\sqrt{\Omega_{k} \Omega_{j}}} \iint_{\xi^{2}+\eta^{2} \leq 1} \frac{T_{B}(\xi, \eta)}{\sqrt{1-\xi^{2}-\eta^{2}}} F_{n k}(\xi, \eta) \\
& \cdot F_{n j}^{*}(\xi, \eta) \tilde{r}_{k j}\left(-\frac{u_{k j} \xi+v_{k j} \eta}{f_{0}}\right) \\
& \cdot e^{-j 2 \pi\left(u_{k j} \xi+v_{k j} \eta\right)} d \xi d \eta,
\end{aligned}
$$

where $k_{B}$ is the Boltzmann's constant, $B_{k, j}$ and $G_{k, j}$ are the noise bandwidth and power gain of the receiving chains (Fig. 2), and $\Omega_{k, j}$ and $F_{n k, j}(\xi, \eta)$ are the equivalent solid angle and normalized radiation voltage patterns of the antennas, assumed to be located in the $X Y$-plane. The director cosines $(\xi, \eta)=$ $(\sin \theta \cos \phi, \sin \theta \sin \phi)$ are defined with respect to the $X$ and $Y$ axes, respectively, and $\tilde{r}_{k j}$ is the so-called fringe-wash function, 


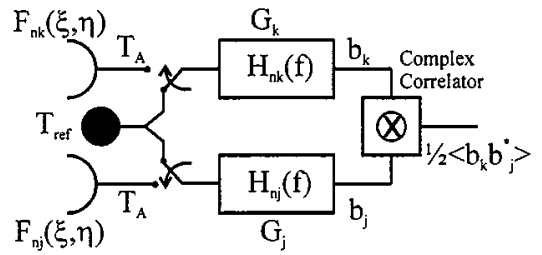

Fig. 2. Pair of antennas and receivers forming a baseline and calibration by noise injection.

which accounts for spatial decorrelation effects and depends on the normalized channel frequency response $H_{n k, j}(f)$ through the relation

$$
\begin{aligned}
\tilde{r}_{k j}(\tau) & =\frac{e^{-j 2 \pi f_{0} \tau}}{\sqrt{B_{k} B_{j}}} \int_{0}^{\infty} H_{n k}(f) H_{n j}^{*}(f) e^{j 2 \pi f \tau} d f \\
& =\frac{1}{\sqrt{B_{k} B_{j}}} \mathrm{~F}^{-1}\left[H_{n k}\left(f+f_{0}\right) H_{n j}^{*}\left(f+f_{0}\right) u\left(f+f_{0}\right)\right]
\end{aligned}
$$

where $f_{0}=c / \lambda_{0}$ is an arbitrary center frequency, $\mathrm{F}^{-1}$ is the inverse Fourier transform operator, and $u()$ is the unity step function. In the ideal case when all the antenna patterns are the same $\left(F_{n j}(\xi, \eta)=F_{n k}(\xi, \eta)\right)$ and spatial decorrelation effects are negligible $\left(\tilde{r}_{k j}(\tau) \approx 1\right)$, the visibility samples and the so-called modified brightness temperature, defined as $T_{B}(\xi, \eta)$. $\left|F_{n}(\xi, \eta)\right|^{2} / \sqrt{1-\xi^{2}-\eta^{2}}$, are related by a Fourier transform. Consequently, since the whole space maps into the area inside the unit circle in the director cosines domain $\xi^{2}+\eta^{2} \leq 1$, the optimum $(u, v)$ spatial frequency sampling is over a hexagonal grid, as obtained from a $Y$ - or $\Delta$-array [3]. In our study, we will assume a $Y$-shaped array of 130 uniformly spaced antennas, as in the original design of the microwave imaging radiometer by aperture synthesis (MIRAS). A 70-antenna version of this instrument has been recently approved for an Earth Explorer mission by the European Space Agency under the name of SMOS [4].

In order to calibrate a single baseline, correlated noise of $k_{B} T_{c} \mathrm{~W} / \mathrm{Hz}$ must be injected at each receiver input (Fig. 2). In this case, (1) reduces to

$$
V_{k j}^{c}=T_{c} \tilde{r}_{k j}(0) .
$$

Thus, provided the correlated noise temperature $T_{c}$ is known, the factor $\tilde{r}_{k j}(0)$, which gives the combined phase and amplitude response of the $k$ and $j$ th receivers, is recovered.

In fact, an InR, e.g., MIRAS, measures the normalized complex cross correlation using 1-bit/2-level digital correlators

$$
\mu_{k j}=\frac{\left\langle b_{k 2}(t) b_{j 2}^{*}(t)\right\rangle}{\sqrt{\left|b_{k 2}(t)\right|^{2}\left|b_{j 2}(t)\right|^{2}}},
$$

and (3) reduces to

$$
\mu_{k j}^{c}=g_{k}^{c} g_{j}^{c} \tilde{r}_{k j}(0) \hat{=} g_{k}^{c} g_{j}^{c} g_{k j} e^{j\left(\theta_{k}-\theta_{j}-\theta_{k j}\right)}
$$

where the term $\tilde{r}_{k j}(0)$ has been split into a nonseparable amplitude $g_{k j}$ and separable $\theta_{k}, \theta_{j}$ and nonseparable $\theta_{k j}$ phase terms.
TABLE I

DIFFERENT RECEIVER ERRORS AND THEIR IMPACT ON $\Delta T$ [5], [6]

\begin{tabular}{cccc}
\hline$\Delta \mathbf{T}$ & $\sigma_{\phi}$ & $\sigma_{\mu}$ & $\Delta \mathbf{T}_{\mathbf{R}}$ \\
\hline $1.0 \mathrm{~K}$ & $1.7^{\circ}$ & $1.610^{-2}$ & $6.3 \mathrm{~K}$ \\
$0.5 \mathrm{~K}$ & $0.83^{\circ}$ & $7.110^{-3}$ & $2.8 \mathrm{~K}$ \\
$0.25 \mathrm{~K}$ & $0.42^{\circ}$ & $3.010^{-3}$ & $1.2 \mathrm{~K}$ \\
$0.12 \mathrm{~K}$ & $0.20^{\circ}$ & $3.010^{-4}$ & $0.12 \mathrm{~K}$ \\
\hline
\end{tabular}

The separable amplitude factors during calibration $\left(g_{k, j}^{c}\right)$ and measurement $\left(g_{k, j}\right)$ are given by [5], [6]

$$
g_{k, j}^{c}=\sqrt{\frac{T_{c}}{T_{c}+T_{R k, j}}} \quad g_{k, j}=\sqrt{\frac{T_{A}}{T_{A}+T_{R k, j}}},
$$

where $T_{R k, j}$ are the receiver noise temperature and $T_{A}$ is the antenna temperature, assumed to be the same for all antennas.

Table I summarizes the requirements for the residual calibration phase/amplitude errors, and the receiver noise temperature [5], [7], in order to achieve a given radiometric resolution $(\Delta T)$. Assuming that all error sources have the same contribution to $\Delta T$, phase and amplitude terms must be known to within $\sim 0.5^{\circ}$ and $\sim 0.5 \%$, and the receiver noise temperature must be known to within $\sim 1.2 \mathrm{~K}$, in order to achieve $\Delta T \approx \sqrt{3} \cdot 0.25 \mathrm{~K}=$ $0.4 \mathrm{~K}$. This value is not the final $\Delta T$, which instead must be computed by taking into account other error sources, such as those generated by the antennas and correlators [8], [9].

The calibration of a multireceiver (multibaseline) InR by means of distributed noise injection (DNI) is an extension of this simple procedure and is explained in detail in [6], [7], and [10]. Receivers are divided into groups of four, as depicted in Fig. 1. Fig. 3(a) and (b) sketches the calibration sequence: a set of noise sources sequentially inject correlated noise to nonoverlapping groups of $2 \times 4=8$ receivers or $3 \times 4=12$ in the central part of the $Y$-array. Note that, with the DNI approach, the largest noise injection networks are: $1: 8$ (along the arms of the array) and 1:12 (at the center). As compared to centralized noise injection (CNI), DNI significantly reduces the mass and volume of the distribution network, and allows much better control of the physical temperature of each network, as well as smaller changes due to mechanical and/or thermal variations.

\section{TheORETICAL ANALYSIS OF THE NOISE DistRIBUtion NETWORK}

In a previous analysis [6], correlated noise at a known equivalent temperature $T_{c}$ was assumed to be injected into each receiver. In practice, this correlated noise must be generated by a common noise source and distributed to all $N$ receivers ( $N=8$, 12). Consequently, the noise distribution network becomes part of the calibration standard and must be well characterized. In this section, the effects of the noise distribution network characteristics on the calibration equations are studied.

Consider the situation in which a noise source at temperature $T_{\text {ref }}$ is connected to port 0 of a passive network at physical temperature $T_{n}$, and the rest of the ports $(1-N)$ are connected to the receivers (Fig. 4). The relationships among the power waves can be obtained from an $S$-parameter analysis. 


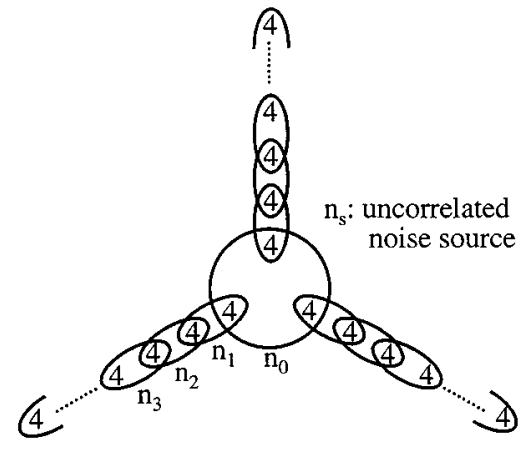

(a)

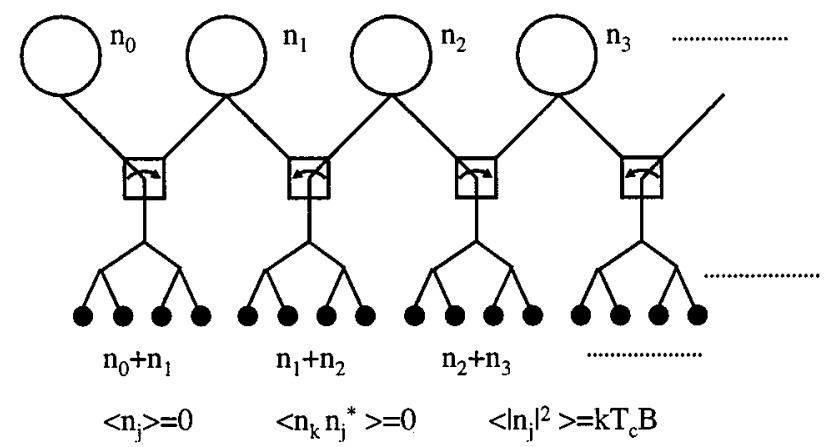

(b)

Fig. 3. (a) Noise injection networks along the $Y$-array. (b) $1: 4$ noise distribution network and position of switches during noise injection. At each position, a different group of eight antennas is formed. When the switch position changes, the groups are shifted by four antennas, allowing the tracking of phase/amplitude relationships along the $Y$-arms.

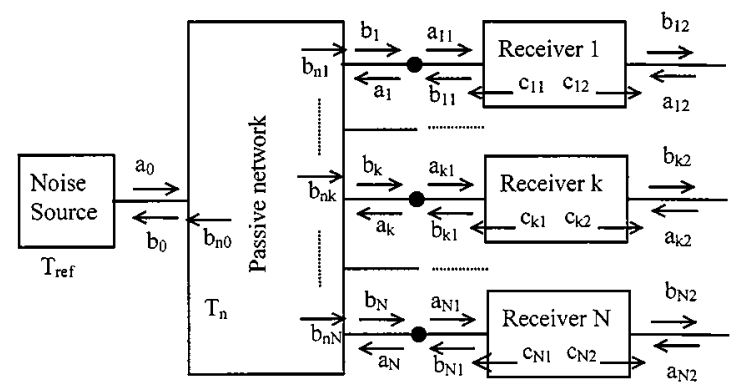

Fig. 4. Noise distribution network and receivers: definition of noise waves.

\section{A. General Noise Theory of Two-Ports}

Receivers connected to the noise injection network are active networks, and their noise behavior may be described in terms of noise waves emerging from both ports $c_{k \cdot 1}, c_{k 2}$ [11]-[13]. These waves are partially correlated and depend on the noise parameters of the receivers. In particular, the mean power of the output wave in the frequency domain is given by [11]-[13] (see also the Appendix)

$$
\left|c_{k 2}\right|^{2}=\left|S_{21}^{(k)}\right|^{2} T_{R k}
$$

where $S_{21}^{(k)}$ is the transmission $S$-parameter of the $k$ th receiver, and $T_{R k}$ is the noise equivalent temperature of the same receiver for a matched source impedance $\left(\Gamma_{s}=0\right)$. On the left-hand side of (7), the averaging operation is implicit in the notation and the factor $1 / 2$ has been omitted, and on the right-hand side, Boltzmann's constant is not explicitly shown.

The noise wave at input $c_{k 1}$ cannot be expressed as a function of the same equivalent noise temperature of the receiver. In fact, it depends on the noise parameters of the active network ( $T_{\min }$, $\Gamma_{\text {opt }}$, and $R_{n}$ ) and on $S_{11}$ [11]. Here, it will be described solely by a different temperature, named $T_{r k}$ (note the lower case " $r$ ")

$$
\left|c_{k 1}\right|^{2}=T_{r k} \text {. }
$$

The complex correlation between $c_{k 1}$ and $c_{k 2}$ depends also on the $S$ - and noise-parameters. Here, it is described by a complex correlation temperature $T_{c k}$ referred to the input

$$
\left\langle c_{k 1} c_{k 2}^{*}\right\rangle=T_{c k} S_{21}^{(k) *} \quad\left\langle c_{k 2} c_{k 1}^{*}\right\rangle=T_{c k}^{*} S_{21}^{(k)} .
$$

Furthermore, receivers will be considered reasonably well matched and without feedback, such that

$$
S_{11}^{(k)}=S_{12}^{(k)}=S_{22}^{(k)}=0 \quad S_{21}^{(k)}=\sqrt{G_{k}} H_{n_{k}}(f)
$$

where $G_{k}$ and $H_{n k}(f)$ are defined in (1) and (2). From the wave definitions shown in Fig. 4, it is apparent that, for the $k$ th port, $b_{k}=a_{k 1}$ and $a_{k}=b_{k 1}$. Therefore, the output of the $k$ th receiver is

$$
b_{k 2}=S_{21}^{(k)} b_{k}+c_{k 2} \text {. }
$$

Taking into account (7), the power output of the $k$ th receiver is $\left|b_{k 2}\right|^{2}=\left|S_{21}^{(k)}\right|^{2}\left(\left|b_{k}\right|^{2}+T_{R k}\right)+S_{21}^{(k)}\left\langle b_{k} c_{k 2}^{*}\right\rangle+S_{21}^{(k) *}\left\langle c_{k 2} b_{k}^{*}\right\rangle$

and the correlation between the outputs of any pair $(k, j)$ of receivers is

$$
\left\langle b_{k 2} b_{j 2}^{*}\right\rangle=S_{21}^{(k)} S_{21}^{(j) *}\left\langle b_{k} b_{j}^{*}\right\rangle+S_{21}^{(k)}\left\langle b_{k} c_{j 2}^{*}\right\rangle+S_{21}^{(j) *}\left\langle c_{k 2} b_{j}^{*}\right\rangle
$$

where the noise generated by each receiver $\left(c_{k 2}\right.$ and $c_{j 2}$ ) has been assumed mutually uncorrelated. The other correlation products are computed in the following section.

From (12) and (13), the normalized cross correlation between the outputs of the $k$ th and $j$ th receivers (4) is

$\mu_{k j}$

$$
\begin{aligned}
= & \frac{S_{21}^{(k)} S_{21}^{(j) *}\left\langle b_{k} b_{j}^{*}\right\rangle+S_{21}^{(k)}\left\langle b_{k} c_{j 2}^{*}\right\rangle+S_{21}^{(j) *}\left\langle c_{k 2} b_{j}^{*}\right\rangle}{\sqrt{\left|S_{21}^{(k)}\right|^{2}\left(\left|b_{k}\right|^{2}+T_{R k}\right)+S_{21}^{(k)}\left\langle b_{k} c_{k 2}^{*}\right\rangle+S_{21}^{(k) *}\left\langle c_{k 2} b_{k}^{*}\right\rangle}} . \\
& \cdot \sqrt{\left|S_{21}^{(j)}\right|^{2}\left(\left|b_{j}\right|^{2}+T_{R j}\right)+S_{21}^{(j)}\left\langle b_{j} c_{j 2}^{*}\right\rangle+S_{21}^{(j)}\left\langle c_{j 2} b_{j}^{*}\right\rangle}
\end{aligned}
$$

In the following section, the terms appearing in (14) are computed.

\section{B. Effect of the Noise Distribution Network}

Using the $S$-parameter formalism, the following equation holds for any network [11]-[13]:

$$
\bar{b}=\overline{\bar{S}} \bar{a}+\bar{b}_{n}
$$

where the column vectors $\bar{b}$ and $\bar{a}$ contain the total outward and inward waves at each port, and $\bar{b}_{n}$ is the column vector of the 
outgoing waves generated by the network itself (Fig. 4). The correlation matrix of the outgoing waves is defined as

$$
\overline{\bar{C}}_{b}=\left[\begin{array}{cccc}
\left|b_{0}\right|^{2} & \left\langle b_{0} b_{1}^{*}\right\rangle & \cdots & \left\langle b_{0} b_{N}^{*}\right\rangle \\
\left\langle b_{1} b_{0}^{*}\right\rangle & \left|b_{1}\right|^{2} & \cdots & \left\langle b_{1} b_{N}^{*}\right\rangle \\
\vdots & \vdots & \ddots & \vdots \\
\left\langle b_{N} b_{0}^{*}\right\rangle & \left\langle b_{N} b_{1}^{*}\right\rangle & \cdots & \left|b_{N}\right|^{2}
\end{array}\right]=\bar{b} \bar{b}^{+} .
$$

The averaging operation is implicit in the notation of the right-hand side of (16) and the superscript " + " stands for "conjugated and transposed." Substituting (15) into (16), the following relationship is easily obtained:

$$
\overline{\bar{C}}_{b}=\overline{\bar{S}} \bar{a} \bar{a}^{+} \overline{\bar{S}}+\overline{\bar{C}}_{n}+\overline{\bar{S}} \bar{a} \bar{b}_{n}^{+}+\bar{b}_{n} \bar{a}^{+} \overline{\bar{S}}^{+}
$$

where $\bar{C}_{n}=\bar{b}_{n} \bar{b}_{n}^{+}$is the correlation matrix of the noise waves generated by the distribution network which, for any passive network, it is related to its $S$-parameters by Bosma's theorem [11]

$$
\overline{\bar{C}}_{n}=T_{n}\left(\overline{\bar{I}}-\overline{\bar{S}} \overline{\bar{S}}^{+}\right)
$$

where $\overline{\bar{I}}$ is the identity matrix and $T_{n}$ is the physical temperature of the network, assumed to be constant.

In the general case, the incident waves $\bar{a}$ are the sum of the waves generated by the terminations (noise source and receivers) and the reflections of the waves $\bar{b}$ going out of the network. If all receivers are well matched, as was already assumed in (10), the incident waves $a_{i}$ are only due to the thermal noise generated by the receivers and by the noise source of port 0 (Fig. 4). Then

$$
\bar{a} \bar{b}_{n}^{+}=\bar{b}_{n} \bar{a}^{+}=0, \quad \bar{a} \bar{a}^{+}=\overline{\bar{T}}
$$

where

$$
\overline{\bar{T}}=\left[\begin{array}{cccc}
T_{\text {ref }} & 0 & \cdots & 0 \\
0 & T_{r 1} & \cdots & 0 \\
\vdots & \vdots & \ddots & \vdots \\
0 & 0 & \cdots & T_{r N}
\end{array}\right]
$$

and where $T_{r k}(k=1, \cdots, N)$ is the equivalent temperature of the wave $c_{k 11}$, as defined in (8), and $T_{\text {ref }}$ is the temperature of the noise source at port 0 (Figs. 3 and 4). Substituting (18)-(20) into (17), it is readily obtained as follows:

$$
\overline{\bar{C}}_{b}=\overline{\bar{S}}\left(\overline{\bar{T}}-T_{n} \overline{\bar{I}}\right) \overline{\bar{S}}^{+}+T_{n} \overline{\bar{I}}
$$

The elements of $\overline{\bar{C}}_{b}$ in (21) can be written in a less compact, but more convenient form to be used in (14)

$$
\begin{aligned}
\left\langle b_{k} b_{j}^{*}\right\rangle & =S_{k 0} S_{j 0}^{*} T_{\text {ref }}+\sum_{i=1}^{N} S_{k i} S_{j i}^{*} T_{r i}-T_{n} \sum_{i=0}^{N} S_{k i} S_{j i}^{*} \\
\left|b_{k}\right|^{2} & =\left|S_{k 0}\right|^{2} T_{\text {ref }}+\sum_{i=1}^{N}\left|S_{k i}\right|^{2} T_{r i}+T_{n}\left(1-\sum_{i=0}^{N}\left|S_{k i}\right|^{2}\right)
\end{aligned}
$$

To obtain the normalized correlation given in (14), it is necessary to compute the cross correlations between the total outward waves $b_{k}$ and the amplifier output waves $c_{k 2}$. From (15), it follows that

$$
b_{k}=\sum_{i=0}^{N} S_{k i} a_{i}+b_{n k} .
$$

Then, since $a_{i}=c_{i 1}$, due to receiver matching

$$
\begin{aligned}
\left\langle b_{k} c_{j 2}^{*}\right\rangle & =\sum_{i=0}^{N} S_{k i}\left\langle a_{i} c_{j 2}^{*}\right\rangle+\left\langle b_{n k} c_{j 2}^{*}\right\rangle \\
& =S_{k j}\left\langle c_{j 1} c_{j 2}^{*}\right\rangle=S_{k j} T_{c j} S_{21}^{(j) *}
\end{aligned}
$$

where $T_{c j}$ is the correlation temperature of the $j$ th receiver defined in (9). In the above expression, it has been taken into account that the noise generated by two receivers is mutually uncorrelated and uncorrelated with respect to the noise generated by the network.

Similarly, the rest of correlations in (14) are given by

$$
\begin{aligned}
& \left\langle c_{k 2} b_{j}^{*}\right\rangle=S_{j k}^{*} T_{c k}^{*} S_{21}^{(k)} \\
& \left\langle b_{k} c_{k 2}^{*}\right\rangle=S_{k k} T_{c k} S_{21}^{(k) *} \\
& \left\langle c_{k 2} b_{k}^{*}\right\rangle=S_{k k}^{*} T_{c k}^{*} S_{21}^{(k)} \\
& \left\langle b_{j} c_{j 2}^{*}\right\rangle=S_{j j} T_{c j} S_{21}^{(j) *} \\
& \left\langle c_{j 2} b_{j}^{*}\right\rangle=S_{j j}^{*} T_{c j} S_{21}^{(j)} .
\end{aligned}
$$

Substituting (22)-(25) into (14), the general expression for the normalized cross correlation is obtained by substituting

$$
\begin{aligned}
\left|b_{k, j 2}\right|^{2}=\left|S_{21}^{(k, j)}\right|^{2}( & \left|S_{k, j 0}\right|^{2} T_{\text {ref }}+\sum_{i=1}^{N}\left|S_{k, j i}\right|^{2} T_{r i} \\
& +T_{n}\left(1-\sum_{i=0}^{N}\left|S_{k, j i}\right|^{2}\right)+T_{R k, j} \\
& \left.+S_{k k, j j} T_{c k, j}+S_{k k, j j}^{*} T_{c k, j}^{*}\right) \\
\left\langle b_{k 2} b_{j 2}^{*}\right\rangle=S_{21}^{(k)} S_{21}^{(j) *} & \left(S_{k 0} S_{j 0}^{*} T_{\text {ref }}+\sum_{i=1}^{N} S_{k i} S_{j i}^{*} T_{r i}\right. \\
-T_{n} \sum_{i=0}^{N} & \left.S_{k i} S_{j i}^{*}+S_{k j} T_{c j}+S_{j k}^{*} T_{c k}^{*}\right)
\end{aligned}
$$

into (4). In the above equations, the first term corresponds to the correlated noise injected into the receivers, while the one containing the factor $T_{n}$ corresponds to the noise generated by the network losses. The fourth term in (26a) corresponds to receivers noise temperature that is added to the noise power being injected. The other terms correspond to the contributions coming from receiver noise coupled through the noise injection network due to the finite isolation. Note that these last terms are very problematic for the calibration since:

- they are not controlled, are difficult to quantify, and are expected to vary with receiver temperature drifts and aging, and

- when receivers are connected to the antennas, the noise radiated by the receivers toward the antenna $T_{r k}$ is collected by other receivers through the antenna coupling $S_{k j}^{\text {ant }}$. 
It should be noted that, from (10), the multiplying term of the normalized cross correlation computed from (4) and (26) can be written as

$$
\frac{S_{21}^{(k)} S_{21}^{(j) *}}{\left|S_{21}^{(k)}\right|\left|S_{21}^{(j)}\right|}=\frac{H_{n k}(f) H_{n j}^{*}(f)}{\left|H_{n k}(f)\right|\left|H_{n j}(f)\right|}
$$

This result is obtained for the frequency-domain description of noise, whereas the quantity measured by the interferometer is the normalized correlation between the time-domain analytic signals. As is briefly described in the Appendix, both descriptions of noise are compatible. In this case, instead of products of frequency-domain functions, frequency integrals of the following type should be used:

$$
\int_{0}^{\infty} H_{n_{k}}(f) S_{k 0}(f) H_{n_{j}}^{*}(f) S_{j 0}^{*}(f) d f
$$

which gives the nonseparable term $g_{k j}$ in (5).

However, if the frequency variation of the $S$-parameters of the noise distribution network is smooth, so that they do not change in the receiver bandwidth, they can be replaced by their complex value at the center frequency. In this case, the factor for representation of the analytic signals (27) is reduced to the fringe-wash function at the origin $\tilde{r}_{k j}(0) \equiv S_{21}^{(k)} S_{21}^{(j) *} /\left|S_{21}^{(k)}\right|\left|S_{21}^{(j)}\right|(2)$.

Finally, if the receivers have input isolators, their input and output noise waves $c_{k 1}$ and $c_{k 2}$ are uncorrelated, thus, $T_{c k}=0$ for all $k$, and all the correlations in (24) and (25) vanish. This issue will be treated in more detail in Section III-A. Furthermore, if the noise distribution network has isolated outputs, i.e., $S_{k j}=0$ for $k \neq j$, then the cross terms (24) and the first term of (25) are null anyway. In this case, (26) simplifies, leading to (29), shown at the bottom of this page, where $\phi_{k 0}$ and $\phi_{j 0}$ are the phases of $S_{k 0}$ and $S_{j 0}$. Note that it requires the network to be phase matched to within $0.5^{\circ}$ [5]. From (29), the separable amplitude error terms are

$$
\begin{aligned}
& g_{k}^{c}=\sqrt{\frac{\left|S_{k 0}\right|^{2}\left(T_{\text {ref }}-T_{n}\right)}{\left|S_{k 0}\right|^{2} T_{\text {ref }}+T_{n}\left(1-\left|S_{k 0}\right|^{2}\right)+T_{R k}}} \\
& g_{j}^{c}=\sqrt{\frac{\left|S_{j 0}\right|^{2}\left(T_{\text {ref }}-T_{n}\right)}{\left|S_{j 0}\right|^{2} T_{\text {ref }}+T_{n}\left(1-\left|S_{j 0}\right|^{2}\right)+T_{R j}}} .
\end{aligned}
$$

Note that, if $T_{n}=0 \mathrm{~K}$ and the network is perfectly symmetrical $\left|S_{k 0}\right|=\left|S_{j 0}\right|$, (30) reduces to the ideal case (6) with $T_{c}=\left|S_{k, j 0}\right| T_{\text {ref. }}$. It must be recalled that, in the preceding analyses, it is implicit that the frequency variation of the noise distribution network is negligible within the useful bandwidth of the receivers. Amplitude calibration consists of extracting $T_{\text {ref }}$ and $T_{R k, j}$ from the set of $g_{k, j}^{c}$ in order to compute the terms $g_{k, j}$ in (6). Note that the accuracy to which $T_{\text {ref }}$ and $T_{R k, j}$ can be determined is limited by the estimation accuracy of the noise introduced by the network [ $T_{n}$ terms of (29)]. Finally, the accuracy of the calibration depends on the accuracy of the knowledge of the physical temperature of the network, the accuracy to which the network $S$-parameters are known before launch, and their drifts during the instrument's life.

\section{NumericAl Simulation OF THE NOISE DistRibution NETWORK}

\section{A. Preliminary Considerations}

At this point, it must be remembered that antenna coupling for the $0.89 \lambda_{0}$ minimum baseline (MIRAS case [4, Fig. 1]) ranges from $\left|S_{k j}^{\text {ant }}\right|=-20$ to $-30 \mathrm{~dB}$, which is worse than the typical isolation of Wilkinson power splitters. Note that, when the receivers are connected to the antennas, as in Fig. 2, an offset term $S_{k j}^{\text {ant }} T_{c k}$ is measured, which is different from the one during calibration [6]. Consequently, in order to satisfy the accuracy required for interferometric arrays [3], [4], an isolator, with an isolation better than $20 \mathrm{~dB}\left(S_{12}^{\text {issol }}+S_{k j}^{\text {ant }} \leq-40 \mathrm{~dB}\right)$, must be inserted between the switch (Fig. 2) and the receiver. The isolator reduces antenna coupling by over $20 \mathrm{~dB}$ and makes this residual offset term negligible. Another function of the isolator is to improve the front-end matching (typically a low-noise amplifier (LNA) is not very well matched), at the expense of a slightly higher noise figure, due to its insertion losses, typically $0.1-0.2 \mathrm{~dB}$. Note that, in order to minimize reflections of amplifiers' outgoing noise waves, it must also be very well matched $(<-25 \mathrm{~dB})$. The tuning to reach the required performance should not be a problem since usually the relative bandwidths allocated for passive observations are very small, i.e., less than $2 \%$ at $L$-band. In addition, since receiver physical temperatures will be controlled to within a few degrees Celsius, it is not expected that their performance will vary significantly.

\section{B. Analysis of Noise Injection Networks}

Preliminary analyses have shown the following.

- Nnonresistive power splitters do not have enough isolation, even with isolators at their outputs, so that $T_{r k, j}$ contributions can be neglected [(8), Fig. 4].

- $1: \mathrm{N}$ isolated power splitters do not have enough isolation either, except for $N=2,3$ (radial-type Wilkinson power splitters) and $N=2$ (fork-type Wilkinson power splitters).

- The isolation resistance in Wilkinson power splitters adds correlated noise at the outputs of the power splitter, which must be accounted for to achieve an accurate calibration.

This preliminary analysis has resulted in the selection of $1: 2$ and $1: 3$ isolated power splitters with isolators at their outputs as the best choice. Hence, further and more accurate analysis is devoted to these kinds of power splitters.

$$
\mu_{k j}^{c}=\tilde{r}_{k j}(0) e^{j\left(\phi_{k 0}-\phi_{j 0}\right)} \frac{\left|S_{k 0}\right|\left|S_{j 0}\right|\left(T_{\mathrm{ref}}-T_{n}\right)}{\sqrt{\left|S_{k 0}\right|^{2} T_{\mathrm{ref}}+T_{n}\left(1-\left|\mathrm{S}_{\mathrm{k0}}\right|^{2}\right)+\mathrm{T}_{\mathrm{Rk}}} \sqrt{\left|S_{j 0}\right|^{2} T_{\mathrm{ref}}+T_{n}\left(1-\left|S_{j 0}\right|^{2}\right)+T_{R j}}}
$$




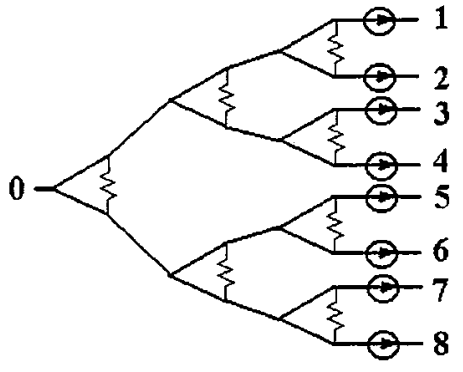

(a)

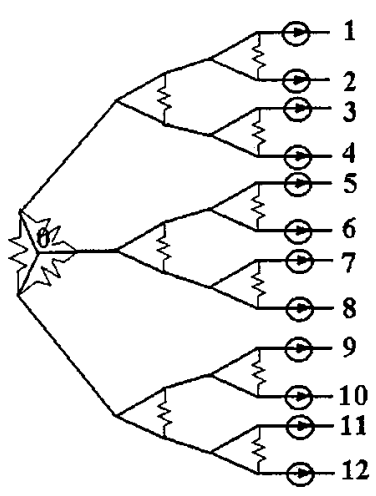

(b)

Fig. 5. Isolated noise injection networks. (a) $1: 8$. (b) $1: 12$.

TABLE II

PARAmeters USED In THE SimUlation OF THE NOISE INJECTION NETWORKS

\begin{tabular}{ll}
\hline Isolators: & $\left|\mathrm{S}_{11}\right|=\left|\mathrm{S}_{22}\right|=-20 \mathrm{~dB},\left|\mathrm{~S}_{21}\right|=0.3 \mathrm{~dB},\left|\mathrm{~S}_{12}\right|=-30 \mathrm{~dB}$ \\
\hline Transmission lines: & $\varepsilon_{\mathrm{r}}=1$, variable attenuation $\alpha \mathrm{dB} / \mathrm{m}$ \\
\hline Frequency range: & $1400 \mathrm{MHz}-1430 \mathrm{MHz}$ \\
\hline
\end{tabular}

The required $1: 8$ and $1: 12$ noise injection networks have been analyzed with MMICAD software. ${ }^{1}$ Note that the $1: 8$ networks correspond to those located along the $Y$-arms, while the $1: 12$ network corresponds to the one injecting noise to the first three antennas of each arm, and to the three additional antennas located in the center for phase restoration purposes. It should be remembered that these networks are formed by grouping 2 or $31: 4(1: 2 / 1: 2)$ power splitters feeding a group of four receivers. The optimum configurations that have been found are $1: 8=1: 2 / 1: 2 / 1: 2$ [see Fig. 5(a)] and $1: 12=1: 3 / 1: 2 / 1: 2$ [see Fig. 5(b)] radial Wilkinson power splitters with isolators at their outputs.

The numerical analysis consisted of:

- evaluation, by means of MMICAD software, of the $S$-parameters for each network topology at the center frequency (1415 MHz) and at the band edges (1400 and $1430 \mathrm{MHz}$ ), for different transmission line attenuation $\alpha$ dBm (Table II);

- computation of the elements of $\overline{\bar{C}}_{n}$ (18) within the protected 1400-1427-MHz bandwith (Table III);

${ }^{1}$ MMICAD is a trademark of Optotek, Kanata, Ont., Canada. [Online]: www.optotek.com

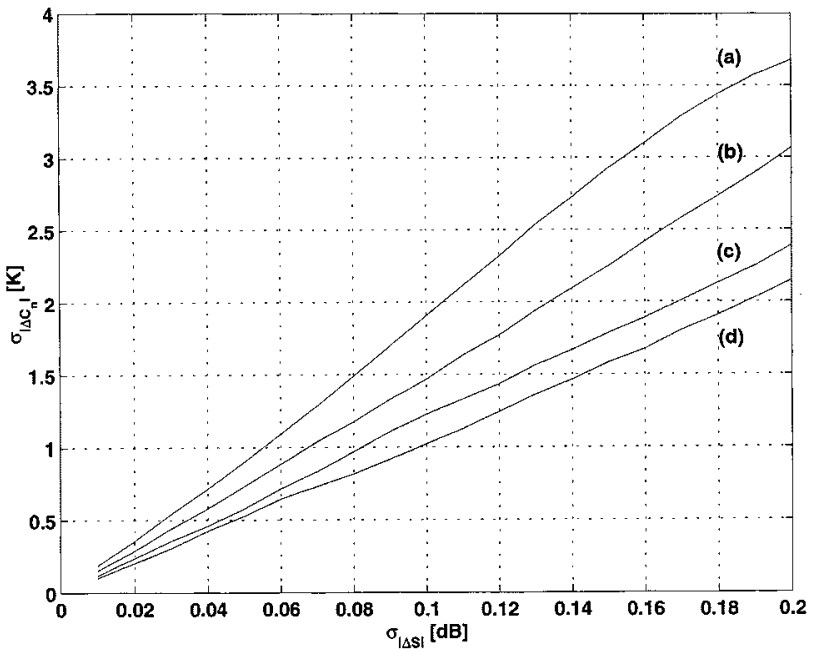

Fig. 6. $\sigma_{C n k j}$ versus $\sigma_{|S|}$ in the measurement of the $S$-parameters of the noise injection network. Physical temperature of the network $=T_{0}=290 \mathrm{~K}$. (a) $1: 8$ without isolators. (b) $1: 12$ without isolators. (c) $1: 8$ with isolators, and (d) $1: 12$ with isolators.

- computation of the error in the estimated values of the elements of $\overline{\bar{C}}_{n}$ (18), for a given random error in the measured $S$-parameters of the network (Rayleigh amplitude error and uniform $(0,2 \pi)$ phase error, as in Fig. 6);

- evaluation of the radiometric accuracy degradation due to the error induced in the estimation of $T_{R k}$ through $\overline{\bar{C}}_{n}$.

Numerical simulations have shown that the variation of the $S$-parameters due to the finite isolation between output ports is negligible within the protected $1400-1427-\mathrm{MHz}$ band, even in the case that one output port is an open circuit (receiver failure): $|\Delta S|<2.10^{-4}$.

The variation of the elements of $\overline{\bar{C}}_{n}$ is even smaller, and can be neglected, and also its effect on $T_{R k, j}$ (Table III).

Fig. 6 shows the mean rms value of the deviation of the $\overline{\bar{C}}_{n}$ 's elements versus the standard deviation of the amplitude of the $S$-parameters. This error adds directly to the estimation of $T_{R k, j}$. Hence, since the maximum accuracy to which the $S$-parameters can be measured is estimated to be on the order of $\sigma_{|\Delta S|}=0.025 \mathrm{~dB}, \overline{\bar{C}}_{n}$ 's elements rms errors will be about $0.30 \mathrm{~K}$ for the $1: 8$ divider, and $0.28 \mathrm{~K}$ for the $1: 12$ divider. Consequently, in order to achieve a calibration accuracy of $0.1 \%(320 \mathrm{~K} \cdot 0.1 \%=0.32 \mathrm{~K}<\Delta T \approx 1 \mathrm{~K}[7])$, $T_{c}=\left|S_{k 0}\right|^{2} \cdot T_{\text {ref }} \geq 320 \mathrm{~K}=1000 \cdot 0.32 \mathrm{~K}$ [numerator of (30)]. This value of the injected noise power is well within the receivers' dynamic range, which guarantees that the receivers will be operating far below saturation. It means that the noise sources driving the $1: 8$ and $1: 12$ power splitters must have an equivalent noise temperature of $2560 \mathrm{~K}=8 \cdot 320 \mathrm{~K}$ $(\mathrm{ENR}=9.5 \mathrm{~dB})$ and $3840 \mathrm{~K}=12 \cdot 320 \mathrm{~K}(\mathrm{ENR}=11.2 \mathrm{~dB})$, respectively.

\section{CONCLUSIONS}

This paper has analyzed in detail the effect of noise injection networks on the calibration of InR's. A principal result is the finding that a large error source during measurement is 
TABLE III

SUMmaRY OF NOISE PARAMETERS OF $1: 8$ AND $1: 12$ NOISE INJECTION NETWORKS. TRANSMISSION-LiNE ATTENUATION: $\alpha \mathrm{dB} / \mathrm{m}$

\begin{tabular}{cccc}
\hline Network type & Element $\overline{\bar{C}}_{n_{k k}}$ & Element $\overline{\bar{C}}_{n_{k j}}$ & Theoretical $S_{k o}$ \\
\hline 1:8 isolated NI network & $0.8833+0.0044 \alpha$ & $-0.1167+0.0044 \alpha$ & $\mathrm{j}(0.3416+0.0065 \alpha)$ \\
$1: 12$ isolated NI network & $0.9222+0.0030 \alpha$ & $-0.0778+0.0030 \alpha$ & $\mathrm{j}(0.2789+0.0055 \alpha)$ \\
\hline
\end{tabular}

the offset induced by the noise radiated by the receivers themselves $\left(T_{r k, j}\right)$ coupled through the antennas. This offset, which differs from the one measured during the calibration process, can be minimized with the insertion of an isolator between the front-end and the switch (isolation $>20 \mathrm{~dB}$ ). The larger isolation that is achieved also simplifies the formulation of the calibration equations, reducing the number of unknowns to two: the physical temperature of the distribution network and its $S$-parameter matrix.

To achieve a residual calibration error of $10^{-3}\left(\Delta T^{\mathrm{cal}}=\right.$ $0.32 \mathrm{~K})$ :

- the physical temperature of the network must be accurately controlled or measured to $\Delta T_{n}<0.5 \mathrm{~K}$;

- the amplitudes of the distribution network's $S$-parameters have to be accurately known, : i.e., $\sigma_{|\Delta S|} \approx 0.025-0.050 \mathrm{~dB}$ for a calibration accuracy on the order of $0.1 \%-0.2 \%$;

- $T_{\text {ref }} \geq N \cdot 320 \mathrm{~K}$ for a $1: N$ network, in order to reasonable neglect the residual error in the estimation of the noise generated by network losses;

- network phase errors add directly to the phase estimation error. Hence, phase unbalance must be kept below $0.5^{\circ}$ or measured within this accuracy;

- an isolator at the receivers' inputs is mandatory to avoid reradiation of correlated noise.

\section{APPENDIX DIFFERENT DESCRIPTIONS OF NOISE}

The $S$-parameters are relationships between complex amplitudes of signals in the frequency domain, thus, this is implicitly the representation of noise when $S$-parameter analysis is used. On the other hand, in the analysis of an interferometer, noise is described by the time-domain analytic signal formalism, which is related to the frequency domain. Some formulas relating both formalisms are used. The interested reader should consult more complete and rigorous expositions in textbooks.

Consider a noise wave represented by its complex amplitude in the frequency domain $A(f)$. Its power in a differential bandwidth $d f$ is

$$
|A|^{2}=k_{B} T_{A} d f
$$

where $T_{A}$ is the equivalent noise temperature and $k_{B}$ is the Boltzmann's constant, which has now been made explicit to preserve the units of power. The two noise signals $B(f)$ and $C(f)$ are now related to $A(f)$ by

$$
B(f)=A(f) S_{b a}(f) \quad C(f)=A(f) S_{c a}(f)
$$

where $S_{b a}$ and $S_{c a}$ are generic $S$-parameters of reflection or transmission, and their power in the bandwidth $d f$ is

$$
\begin{aligned}
& |B|^{2}=|A|^{2}\left|S_{b a}\right|^{2}=k_{B} T_{A}\left|S_{b a}\right|^{2} d f \\
& |C|^{2}=|A|^{2}\left|S_{c a}\right|^{2}=k_{B} T_{A}\left|S_{c a}\right|^{2} d f
\end{aligned}
$$

and their cross correlation in the same bandwidth is

$$
\left\langle B C^{*}\right\rangle=|A|^{2} S_{b a} S_{c a}^{*}=k_{B} T_{A} S_{b a} S_{c a}^{*} d f
$$

If the above signals are described by their analytic representations $a(t), b(t)$, and $c(t)$, assuming that $a(t)$ is wide-band (white noise), the total average power and cross correlation of the last two are

$$
\begin{gathered}
|b|^{2}=k_{B} T_{A} \int_{0}^{\infty}\left|S_{b a}\right|^{2} d f \\
|c|^{2}=k_{B} T_{A} \int_{0}^{\infty}\left|S_{c a}\right|^{2} d f \\
\left\langle b c^{*}\right\rangle=k_{B} T_{A} \int_{0}^{\infty} S_{b a} S_{c a}^{*} d f .
\end{gathered}
$$

Thus, to pass from the frequency domain to the analytic signal description, it suffices to substitute the frequency-domain functions $S_{i j}(f)$ by the above integrals.

\section{REFERENCES}

[1] A. R. Thompson, J. M. Moran, and G. W. Swenson, Interferometry and Synthesis in Radio Astronomy. New York: Wiley, 1986.

[2] C. S. Ruf, C. T. Swift, A. B. Tanner, and D. M. Levine, "Interferometric synthetic aperture radiometry for the remote sensing of the Earth," IEEE Trans. Geosci. Remote Sensing, vol. 26, pp. 597-611, Sept. 1988.

[3] A. Camps, J. Bará, I. Corbella, and F. Torres, "The processing of hexagonally sampled signals with standard rectangular techniques: Application to 2-D large aperture synthesis interferometric radiometers," IEEE Trans. Geosci. Remote Sensing, vol. 35, pp. 183-190, Jan. 1997.

[4] M. Martín-Neira and J. M. Goutoule, "MIRAS-A two-dimensional aperture-synthesis radiometer for soil-moisture and ocean salinity observations," ESA Bull., no. 92, pp. 95-104, Nov. 1997.

[5] F. Torres, A. Camps, J. Bará, and I. Corbella, "Impact of receiver errors on the radiometric resolution of large 2D aperture synthesis radiometers: Study applied to MIRAS," Radio Sci., vol. 32, no. 2, Mar.-Apr. 1997.

[6] F. Torres, A. Camps, J. Bará, I. Corbella, and R. Ferrero, "On-board phase and modulus calibration of large aperture synthesis radiometers: Study applied to MIRAS," IEEE Trans. Geosci. Remote Sensing, vol. 34, pp. 1000-1009, July 1996.

[7] A. Camps, "Application of interferometric radiometry to Earth observation,” Ph.D. dissertation, Dept. Telecommun. Eng., Univ. Politècnica de Catalunya, Barcelona, Spain, 1996.

[8] A. Camps, J. Bará, F. Torres, I. Corbella, and J. Romeu, "Impact of antenna errors on the radiometric accuracy of large aperture synthesis radiometers: Study applied to MIRAS," Radio Sci., vol. 32, no. 2, pp. 657-668, Mar.-Apr. 1997.

[9] A. Camps, F. Torres, I. Corbella, J. Bará, and J. A. Lluch, "Threshold and timing errors of 1 bit/ 2 level digital correlators in Earth observation synthetic aperture radiometry," Electron. Lett., vol. 33, no. 9, pp. 813-821, Apr. 1997. 
[10] I. Corbella, F. Torres, A. Camps, and J. Bará, "A new calibration technique for interferometric radiometers," in Proc. SPIE Conf. Sensors, Syst., Next-Generation Satellites IV, Barcelona, Spain, Sept. 21-24, 1998, pp. 359-366.

[11] H. Bosma, "On the theory of linear noisy systems," Philips Res., Rep. Supp. 10, 1967

[12] S. Wedge and D. Rutledge, "Wave techniques for noise modeling and measurement," IEEE Trans. Microwave Theory Tech., vol. 40, pp. 2004-2012, Nov. 1992.

[13] — "Noise waves and passive linear multiports," IEEE Microwave Guided Wave Lett., vol. 1, pp. 117-119, May 1991.

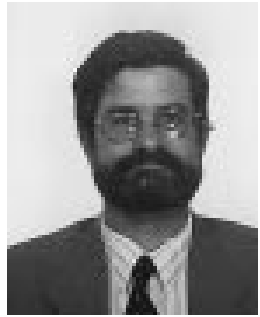

Ignasi Corbella (M'99) was born in Barcelona, Spain, in 1955. He received the engineer and doctor engineer degrees in telecommunication engineering from the Universitat Politècnica de Catalunya (UPC), Barcelona, Spain, in 1977 and 1983, respectively.

In 1976, he joined the School of Telecommunication Engineering, Barcelona, Spain, as a Research Assistant in the Microwave Laboratory, were he was involved with passive microwave integrated-circuit design and characterization. He became an Assistant Professor in 1982, Associate Professor in 1986, and Professor in 1993. He is currently teaching microwaves at an undergraduate level at the UPC. During the 1998-1999 academic year, he was with the NOAA/Environmental Technology Laboratory, Boulder, CO, as a Guest Researcher, where he developed methods for radiometer calibration and data analysis. His research work in the Department of Signal Theory and Communications, UPC, includes microwave airborne and satellite radiometry and microwave system design.

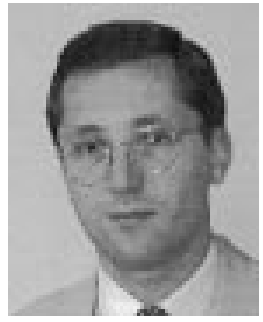

Adriano Camps (S'91-A'97) received the telecommunications engineer degree and Ph.D. degree in telecommunications engineering from the Polytechnic University of Catalonia, Barcelona, Spain, in 1992 and 1996, respectively. His Ph.D. dissertation topic concerned the passive remote sensing of the Earth by means of interferometric radiometry, focusing on the SMOS/MIRAS $L$-band InR, a mission currently approved by the European Space Agency.

From 1991 to 1992, he was with the Ecole Nationale Supérieure des Télécommunications de Bretagne, Bretagne, France. In 1993, he joined the Electromagnetics and Photonics Engineering Group, Department of Signal Theory and Communications, Universitat Politècnica de Catalunya (UPC), as an Assistant Professor, and has been an Associate Professor since 1997. During 1999, he was on sabbatical leave at the Microwave Remote Sensing Laboratory, University of Massachusetts. His research interests are focused on passive and active microwave remote sensing.

Dr. Camps is member of the Spanish Association of Telecommunication Engineers. He was the recipient of a Erasmus Fellowship. In 1993, he received the second Spanish National Prize of University Studies, and in 1997, he received the INDRA Award for the best Ph.D. in remote sensing.

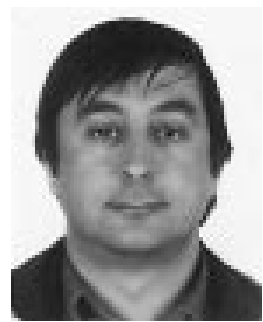

Francesc Torres was born in Ibiza, Spain, in 1962. $\mathrm{He}$ received the ingeniero and doctor ingeniero degree in telecommunication engineering from the Universitat Politècnica de Catalunya (UPC), Barcelona, Spain, in 1988 and 1992, respectively.

From 1988 to 1989, he was a Research Assistant in the RF System Division, European Space Agency, Noordwijk, The Netherlands, where his research was devoted to microwave device testing and characterization. In 1989, he joined the Antenna-MicrowaveRadar Group, UPC, where he is currently a Professor. His main research interests are focused on the design and testing of microwave systems and subsystems. He is currently engaged in research on INR's devoted to Earth observation.

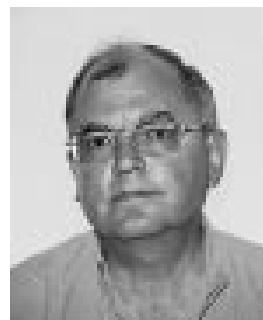

Javier Bará was born on September 30, 1944. He received the Sc.M. and Ph.D. degrees from Brown University, Providence, RI, in 1968 and 1972, respectively, both in electrical engineering.

Since 1972, he has been a Professor at the Universitat Politècnica de Catalunya (UPC), Barcelona, Spain, where he has held several posts of academic responsibility, such as Associate School Dean, Dean, and Department Director.

His research interests have been in the field of microwaves (ferrites, integrated circuits, satellite communications, industrial heating, and drying processes) and is currently involved in projects in nonguided optical communications in the near-infrared and interferometric radiometry for remote sensing of the Earth. 